\title{
Nonlinear Elliptic Equations Involving Critical Sobolev Exponents: Asymptotic Analysis via Methods of Epi-Convergence
}

\author{
C. Bandle. and A. Brillard
}

\begin{abstract}
We study the minimizers of two functionals involving critical Sobolev exponents, and whose Euler equations lead to nonlinear boundary value problems. We first employ classical methods to obtain estimates. We then rephrase the problems in a more abstract functional analytical setting. We use epi-convergence arguments in order to describe the behaviour of the minimizers.

Keywords: Calculus of variations, minimizing sequences, critical Sobolev exponents, epi-convergence
\end{abstract}

AMS subject classification: $35 \mathrm{~B} 05,35 \mathrm{~J} 65,49 \mathrm{~J} 45$

\section{Introduction}

Consider the variational problem

$$
\inf _{u \in K(\Omega)} \int_{\Omega}|\nabla u|^{2} d x=: C_{2} \text {. }
$$

where $\Omega$ is a bounded, smooth and open subset of $\mathbb{R}^{N}(N \geq 3), K(\Omega)$ is defined as

$$
K(\Omega)=\left\{\left.u \in H_{0}^{1}(\Omega)\left|\int_{\Omega}\right| u\right|^{2^{\bullet}} d x=1\right\}
$$

and $2^{*}$ denotes the critical Sobolev exponent $2^{*}=2 N /(N-2)$. It is well known (see, for example, [5]) that the minimum value $C_{2}$. is never achieved. Moreover, $C_{2}$. is independent of $\Omega$ and has the constant value

$$
C_{2} \cdot=\pi N(N-2)\left(\frac{\Gamma(N / 2)}{\Gamma(N)}\right)^{2 / N} .
$$

According to a result of P. L. Lions [12: Lemma I.1], every minimizing sequence of problem (P1) contains a subsequence $\left(u_{k}\right)_{k}$ which concentrates at some point $x_{0} \in \Omega$, that is the sequence $\left(\left|\nabla u_{k}\right|\right)_{k}$ converges to $\delta_{x_{0}}$, weakly in the sense of measures.

C. Bandle: Univ. Basel, Math. Institut, Rheinsprung 21, CH-4051 Basel

A. Brillard: Univ. de Haute-Alsace, 4 Rue des Frères Lumière, F-68093 Mulhouse

ISSN 0232-2064 / \$2.50 (c) Heldermann Verlag Berlin 
Besides of the problem (P1), we shall consider two perturbations, the behaviour of which differs considerably from that of problem (P1). The first one is

$$
\inf _{u \in K_{*}(\Omega)} \int_{\Omega}|\nabla u|^{2} d x=: C_{\varepsilon 2}(\Omega)
$$

where $\varepsilon>0$ and the set $K_{\varepsilon}(\Omega)$ is defined as

$$
K_{\varepsilon}(\Omega)=\left\{\left.u \in \cdot H_{0}^{1}(\Omega)\left|\int_{\Omega}\right| u\right|^{2^{\bullet}-\varepsilon} d x=1\right\}
$$

Since, for every $\varepsilon>0$, the embedding $H_{0}^{1}(\Omega) \hookrightarrow L^{2^{*}-\varepsilon}(\Omega)$ is compact, there exists at least one solution $u_{e 2}$ of problem (P2) $)_{e}$. It can be proved by standard arguments that $u_{\varepsilon 2}$, which can be chosen to be positive in $\Omega$, is the solution of the problem

$$
\begin{aligned}
& -\Delta u_{\varepsilon 2}=\left(\int_{\Omega}\left|\nabla u_{\varepsilon 2}\right|^{2} d x\right) u_{\varepsilon 2}^{2^{*}-\varepsilon-1} \quad \text { in } \Omega \\
& \int_{\Omega}\left|u_{\varepsilon 2}\right|^{2^{*}-\varepsilon} d x=1 \quad\left(u_{\varepsilon 2} \in H_{0}^{1}(\Omega)\right) .
\end{aligned}
$$

The second perturbed problem considered in this study is

(P3)e $\inf _{u \in K(\Omega)}\left(\int_{\Omega}|\nabla u|^{2} d x-\varepsilon \int_{\Omega} u^{2} d x\right)=: C_{e 3}(\Omega)$

where $K(\Omega)$ again is defined in (1). According to [5: Lemma 1.6] (see also [16: Lemma $2.2 / \mathrm{p} .159])$ and the result of P.L. Lions mentioned above, $C_{\epsilon 3}(\Omega)$ is attained if and only if $C_{e 3}(\Omega)<C_{2^{*}}$. As it is shown in [5: Theorems 1.1 and 1.2], this is the case if

(i) $N \geq 4$ and $\varepsilon \in\left(0, \lambda_{1}\right)$, where $\lambda_{1}$ is the first eigenvalue of $-\Delta$ in $\Omega$

(ii) $N=3$ and $\varepsilon \in\left(\lambda^{*}, \lambda_{1}\right)$, for some positive $\lambda^{*}$ depending on the domain [3].

In these cases, the minimizers $u_{e 3}$ of problem (P3) $)_{e}$ which can always be taken to be positive, belong to $K(\Omega)$ and satisfy

$$
-\Delta u_{\varepsilon 3}=\left(\int_{\Omega}\left|\nabla u_{\varepsilon 3}\right|^{2} d x-\varepsilon \int_{\Omega} u_{e 3}^{2} d x\right) u_{\varepsilon 3}^{2^{*}-1}+\varepsilon u_{\varepsilon 3} \quad \text { in } \Omega .
$$

If there is no minimizer of problem $(\mathrm{P} 3)_{\varepsilon}$, we consider a minimizing sequence and denote by $u_{e 3}$ a member of this sequence such that

$$
\begin{array}{ll}
\int_{\Omega}\left|\nabla u_{e 3}\right|^{2} d x-\varepsilon \int_{\Omega} u_{e 3}^{2} d x \leq C_{e 3}(\Omega)+o_{\varepsilon} \\
\int_{\Omega}\left|u_{e 3}\right|^{2^{*}} d x=1 \quad \text { for every } \varepsilon>0
\end{array}
$$


where $o_{\varepsilon}$ satisfies $\lim _{\varepsilon \rightarrow 0} o_{\varepsilon}=0$. In the sequel, $u_{\varepsilon 3}$ will be called an $o_{\varepsilon}$-minimizer. We shall also use the notation $o_{\varepsilon}$ for every quantity converging to 0 when $\varepsilon \rightarrow 0$.

The main purpose of this paper is to study the asymptotic behaviour of the sequences $\left(u_{e 2}\right)_{\varepsilon}$ and $\left(u_{e 3}\right)_{e}$, as $\varepsilon \rightarrow 0$. We start with a discussion of the quantities $C_{e 2}(\Omega)$ and $C_{e 3}(\Omega)$. It is well known that $C_{e k}(\Omega) \rightarrow C_{2}$. as $\varepsilon \rightarrow 0$. We shall estimate the rate of convergence of these sequences in terms of the geometry of $\Omega$. We then translate our results into the language of epi-convergence. This requires that the variational problems $(\mathrm{P} 2)_{e}$ and $(\mathrm{P} 3)_{e}$ are put in an appropriate abstract setting. In the last section, we use the epi-convergence in order to determine the asymptotic shape of $\left(u_{e 2}\right)_{e}$ and $\left(u_{e 3}\right)_{e}$.

\section{Qualitative properties of the minimizing sequences}

2.1 Qualitative properties of the quantities $\dot{C}_{c 2}(\Omega)$ and $C_{c 3}(\Omega)$. The goal of this section is to describe some properties of the quantities $C_{e 2}(\Omega)$ and $C_{e 3}(\Omega)$, in particular their behaviour as $\varepsilon \rightarrow 0$. For the reader's convenience we recall the following

Lemma 2.1: For every bounded subset $\Omega$ of $\mathbb{R}^{N}$, we have

(i) $\lim _{e \rightarrow 0} C_{e 2}(\Omega)=C_{2}$.

(ii) $\lim _{\varepsilon \rightarrow 0} C_{e 3}(\Omega)=C_{2^{*}}$.

Proof: (i) Let $\left(u_{\delta}\right)_{\delta} \subset K(\Omega)$ be a minimizing sequence for problem (P1), that is

$$
\int_{\Omega}\left|\nabla u_{\delta}\right|^{2} d x \leq C_{2} \cdot+o_{\delta}
$$

with $\lim _{\delta \rightarrow 0} o_{\delta}=0$. Set $u_{e \delta}=u_{\delta} /\left\|u_{\delta}\right\|_{L^{2 \cdot-}}$. Then $u_{\varepsilon \delta}$.belongs to $K_{\varepsilon}(\Omega)$ and satisfies

$$
C_{e 2}(\Omega) \leq \int_{\Omega}\left|\nabla u_{e \delta}\right|^{2} d x=\left(C_{2} \cdot+o_{\delta}\right) /\left\|u_{\delta}\right\|_{L^{2 *-\varepsilon}}^{2}
$$

Letting $\varepsilon \rightarrow 0$ and using Lebesgue's dominated convergence theorem, we get the estimation $\lim \sup _{\varepsilon \rightarrow 0} C_{\varepsilon 2}(\Omega) \leq C_{2} \cdot+o_{\delta}$.

Consider now a minimizer $u_{\varepsilon 2}$ of problem (P2) $)_{e}$. Then $u_{\varepsilon 2} /\left\|u_{\varepsilon 2}\right\|_{L^{2} \cdot}$ belongs to $K(\Omega)$ and satisfies

$$
\left\|u_{e 2}\right\|_{L^{2 \cdot}}^{2} C_{2^{\bullet}} \leq \int_{\Omega}\left|\nabla u_{\varepsilon 2}^{\prime}\right|^{2} d x \doteq C_{\varepsilon 2}(\Omega) .
$$

Hölder's inequality implies that

$$
1=\int_{\Omega} u_{e 2}^{2^{*}-\varepsilon} d x \leq\left(\int_{\Omega} u_{e 2}^{2^{*}} d x\right)^{\left(2^{*}-e\right) / 2^{*}}\left(\int_{\Omega} 1 d x\right)^{e / 2^{*}}
$$

Whence

$$
C_{2} \cdot \leq C_{e 2}(\Omega)(\operatorname{meas} \Omega)^{2 e /\left(2^{\bullet}-\varepsilon\right) 2^{*}}
$$

Finally

$$
C_{2 \cdot} \cdot \leq \liminf _{e \rightarrow 0} C_{e 2}(\Omega) \leq \underset{e \rightarrow 0}{\limsup } C_{e 2}(\Omega) \leq C_{2 \cdot}+o_{\delta}
$$


Since $o_{\delta}$ is an arbitrary small number, this establishes the first assertion. we get

(ii) Let $\left(u_{\delta}\right)_{\delta} \subset K(\Omega)$ be as before. Using $u_{\delta}$ as trial function for problem (P3)

$$
C_{e 3}(\Omega) \leq C_{2^{*}}+o_{\delta}-\varepsilon \int_{\Omega} u_{\delta}^{2} d x .
$$

Moreover, by Hölder's inequality, we have for every $u$ in $K(\Omega)$

$$
C_{2^{*}} \leq \int_{\Omega}|\nabla u|^{2} d x-\varepsilon \int_{\Omega} u^{2} d x+\varepsilon(\operatorname{meas} \Omega)^{\left(2^{*}-2\right) / 2^{*}}
$$

whence

$$
C_{2^{\bullet}} \leq C_{\varepsilon 3}(\Omega)+\varepsilon(\operatorname{meas} \Omega)^{\left(2^{\bullet}-2\right) / 2^{\bullet}}
$$

This implies

$$
C_{2^{*}} \leq \liminf _{\varepsilon \rightarrow 0} C_{e 3}(\Omega) \leq \underset{\varepsilon \rightarrow 0}{\limsup } C_{e 3}(\Omega) \leq C_{2^{*}}+o_{\delta}
$$

The proof is now obvious

Remark 2.2: Lemma 2.1 implies that if $u_{\varepsilon 2}$ is a minimizer of problem $(\mathrm{P} 2)_{e}$, the function $w_{\varepsilon}=u_{\varepsilon 2} /\left\|u_{\varepsilon 2}\right\|_{L^{2 \cdot}}(\Omega)$ is an $o_{\varepsilon}$-minimizer of problem (P3) $)_{\varepsilon}$. Indeed, the sequence $\left(u_{e 2}\right)_{e}$ is bounded in $H_{0}^{1}(\Omega)$ and $\lim _{\varepsilon \rightarrow 0} \int_{\Omega}\left|\nabla w_{\varepsilon}\right|^{2} d x=C_{2^{*}}$. Hence

$$
\limsup _{\varepsilon \rightarrow 0}\left(\int_{\Omega}\left|\nabla w_{\varepsilon}\right|^{2} d x-\varepsilon \int_{\Omega} w_{\varepsilon}^{2} d x\right) \leq C_{2^{*}}
$$

In constrast to $C_{2^{*}}$, the quantities $C_{e 2}(\Omega)$ and $C_{e 3}(\Omega)$ depend on the geometry of $\Omega$. Let us list some monotonicity properties of these quantities, which are immediate consequences of their definition:

(i) $C_{\varepsilon 2}\left(\Omega_{1}\right) \leq C_{\varepsilon 2}\left(\Omega_{0}\right)$ and $C_{\varepsilon 3}\left(\Omega_{1}\right) \leq C_{\varepsilon 3}\left(\Omega_{0}\right)$ for $\Omega_{0} \subseteq \Omega_{1}$

(ii) $C_{\varepsilon^{\prime} 3}(\Omega) \geq C_{\varepsilon 3}(\Omega) \quad$ for $0<\varepsilon^{\prime} \leq \varepsilon$.

With the help of rearrangement techniques, we can compare the functionals in $\Omega$ to the corresponding ones in the ball $\Omega^{*}=B\left(0, R^{*}\right):=\left\{|x|<R^{*}\right\}$ of the same volume as $\Omega$. Indeed, we have [2: Chapter 2, Sections 1.1 and 1.2]

(iii) $C_{e 2}(\Omega) \geq C_{e 2}\left(\Omega^{*}\right)$ and $C_{e 3}(\Omega) \geq C_{e 3}\left(\Omega^{*}\right)$.

Next, we use the techniques of harmonic transplantation [2: Chapter 3, Section 4.1] to estimate the quantities $C_{e 2}(\Omega)$ and $C_{e 3}(\Omega)$ from above. For this purpose, we consider the Green function of the Laplace operator in $\Omega$, vanishing at the boundary $\partial \Omega$. It can be represented as

$$
G_{\Omega}(x, y)=\frac{1}{(N-2) \sigma_{N}}\left(\frac{1}{|x-y|^{N-2}}-H(x, y)\right)
$$


where $\sigma_{N}$ is the area of the unit sphere of $\mathbb{R}^{N}\left(\sigma_{N}=\frac{2 \pi^{N / 2}}{\Gamma(N / 2)}\right)$ and $H(\cdot, y)$ is a harmonic function. The quantity

$$
\dot{R}=\max _{x \in \Omega} H^{-1 /(N-2)}(x, x)=H^{-1 /(N-2)}\left(x_{0}, x_{0}\right)
$$

is called the harmonic radius of $\Omega$. Denote by $\dot{\Omega}$ the ball $B(0, \dot{R})$. Now let $u_{\varepsilon 2}$ be a minimizer for $C_{\varepsilon 2}(\dot{\Omega})$. By a rearrangement argument, we can always assume that $u_{\varepsilon 2}=u_{\varepsilon 2}(|x|)>0$. Hence the level surfaces of $u_{\varepsilon 2}(|x|)$ and $G_{\dot{\Omega}}(x, 0)$ coincide and we can set

$$
u_{\varepsilon 2}(|x|)=\hat{u}_{e 2}\left(G_{\dot{\Omega}}(x, 0)\right) \text {. }
$$

Let $U_{e 2}(x)=\hat{u}_{\varepsilon 2}\left(G_{\Omega}\left(x, x_{0}\right)\right)$ be its transplantation into $\Omega$. Then [2: Chapter 3, Section 4.1]

$$
\int_{\dot{\mathbf{\Omega}}}\left|\nabla u_{e 2}\right|^{2} d x=\int_{\Omega}\left|\nabla U_{e 2}\right|^{2} d x \quad \text { and } \quad \int_{\dot{\Omega}} u_{e 2}^{p} d x \leq \int_{\Omega} U_{e 2}^{p} d x \quad(p \geq 1) .
$$

Consequently, $\left\|u_{e 2}\right\|_{L^{2^{*}-\varepsilon}(\dot{\Omega})}=1$ implies $\left\|U_{e 2}\right\|_{L^{2^{*}-\varepsilon^{\prime}(\Omega)}} \geq 1$, whence

$$
C_{e 2}(\Omega) \leq\left\|U_{e 2}\right\|_{L^{2^{*}-e^{\prime}(\Omega)}}^{-2} \int_{\Omega}\left|\nabla U_{e 2}\right|^{2} d x \leq \int_{\dot{\Omega}}\left|\nabla u_{e 2}\right|^{2} d x=C_{\varepsilon 2}(\dot{\Omega}) .
$$

In summary

$$
\text { (iv) } C_{e 2}(\Omega) \leq C_{e 2}(\dot{\Omega})
$$

Similarly, we prove

$$
\text { (v) } C_{\varepsilon 3}(\Omega) \leq C_{\epsilon 3}(\dot{\Omega}) \text {. }
$$

These observations lead to the following

Lemma 2.3: Put $\beta=R^{*} / \dot{R}$. Then

(i) $C_{\varepsilon 2}\left(\Omega^{*}\right) \leq C_{\varepsilon 2}(\Omega) \leq C_{\varepsilon 2}\left(\Omega^{*}\right) \beta^{2-N+2 N /\left(2^{*}-e\right)}$.

(ii) $C_{e 3}\left(\Omega^{*}\right) \leq C_{e 3}(\Omega) \leq C_{\left(\beta^{2} e\right) 3}\left(\Omega^{*}\right) \beta^{2-N+2 N / 2^{*}}$.

Proof: (i) Let $u_{e 2}$ be a minimizer of problem (P2) in $\Omega^{*}$, and set $v_{\varepsilon 2}(x)=u_{\varepsilon 2}(\beta x)$. We have

$$
\left\|v_{e 2}\right\|_{L^{2^{*}-\epsilon}(\dot{\Omega})}^{2^{*}}=\int_{\dot{\Omega}} u_{e 2}^{2^{*}-e}(\beta x) d x=\beta^{-N} .
$$

Taking $v_{\varepsilon 2} /\left\|v_{e 2}\right\|_{L^{2^{*}-\varepsilon}(\dot{\Omega})}$ as a trial function for the variational characterization of $C_{e 2}(\dot{\Omega})$ we obtain

$$
C_{e 2}(\dot{\Omega}) \leq\left\|v_{e 2}\right\|_{L^{2^{*}-\bullet(\dot{\Omega})}}^{-2} \int_{\dot{\Omega}}\left|\nabla v_{\varepsilon 2}\right|^{2} d x=C_{e 2}\left(\Omega^{*}\right) \beta^{2-N+2 N /\left(2^{*}-\varepsilon\right)} .
$$

The same argument shows that the inequality with the reversed sign is also true. This together with statements (iv) and (iii) completes the proof of the first assertion. 
(ii) Let $u_{e 3}$ be an $o_{e}$-minimizer of problem $(\mathrm{P} 3)_{e}$ in the ball $\Omega^{*}$. Then $w_{e 3}(x)=$ $\beta^{N / 2^{*}} u_{e 3}(\beta x)$ belongs to $K(\dot{\Omega})$ and $w_{\varepsilon 3}$ can be taken as trial function for problem (P3) in $\dot{\Omega}$, with $\gamma:=\varepsilon \beta^{2}$. Hence

$$
\begin{aligned}
C_{\gamma^{3}}(\dot{\Omega}) & \leq \int_{\dot{\Omega}}\left|\nabla w_{\varepsilon 3}\right|^{2} d x-\gamma \int_{\dot{\Omega}} w_{\varepsilon 3}^{2} d x \\
& \leq \beta^{2-N+2 N / 2^{*}} \int_{\Omega^{*}}\left|\nabla u_{e 3}\right|^{2} d x-\gamma \beta^{-N+2 N / 2^{*}} \int_{\Omega^{*}} u_{\varepsilon 3}^{2} d x \\
& \leq \beta^{2-N+2 N / 2^{*}}\left(C_{e 3}^{\cdot}\left(\Omega^{*}\right)+o_{\varepsilon}\right) .
\end{aligned}
$$

Since $o_{e}$ is an arbitrary small number, $C_{\gamma 3}(\dot{\Omega}) \leq \beta^{2-N+2 N / 2^{*}} C_{e 3}\left(\Omega^{*}\right)$. This completes the proof

2.2 Qualitative properties of the minimizing sequences $\left(u_{\varepsilon 2}\right)_{\varepsilon}$ and $\left(u_{\varepsilon 3}\right)_{\varepsilon}$. We start with the following result which will be proved for the sake of completeness.

Lemma 2.4: Suppose that $u_{\varepsilon 2}$ is a solution of the minimization problem $(\mathrm{P} 2)_{e}$. Then the sequence $\left(u_{e 2}\right)_{e}$ converges to 0 in the weak topology of $H_{0}^{1}(\Omega)$.

Proof: In view of Lemma $2.1 /(\mathrm{i})$, the sequence $\left(u_{\varepsilon 2}\right)_{\varepsilon}$ possesses at least one limit point $u^{*}$ in the weak topology of $H_{0}^{1}(\Omega)$. We write

$$
\begin{aligned}
\int_{\Omega}\left|\nabla u_{e 2}\right|^{2} d x= & \int_{\Omega}\left|\nabla u^{*}\right|^{2} d x+\int_{\Omega}\left|\nabla\left(u_{\varepsilon 2}-u^{*}\right)\right|^{2} d x \\
& +2 \int_{\Omega} \nabla u^{*} \nabla\left(u_{\varepsilon 2}-u^{*}\right) d x .
\end{aligned}
$$

By the Sobolev inequality,

$$
\begin{aligned}
\int_{\Omega}\left|\nabla u_{e 2}\right|^{2} d x \geq & C_{2^{*}}\left(\int_{\Omega}\left|u^{*}\right|^{2^{*}} d x\right)^{2 / 2^{*}} \\
& +C_{e 2}(\Omega)\left(\int_{\Omega}\left|u_{\varepsilon 2}-\dot{u}^{*}\right|^{2^{*}-\varepsilon} d x\right)^{2 /\left(2^{*}-\varepsilon\right)}+o_{\varepsilon}
\end{aligned}
$$

We recall the following result established in [4]:

Assume that a sequence $\left(f_{k}\right)_{k}$ converges to $f$ in the weak topology of $L^{q}(\Omega) \quad(q \in$ $[1,+\infty)$ ) and almost everywhere in $\Omega$. Then

$$
\lim _{k \rightarrow+\infty}\left(\int_{\Omega}\left|f_{k}\right|^{q} d x-\int_{\Omega}\left|f_{k}-f\right|^{q} d x\right)=\int_{\Omega}|f|^{q} d x
$$

which can be extended in the present case in the following way (see [6]):

Assume that a. sequence $\left(f_{\varepsilon}\right)_{e}$ converges in the weak topology of $H_{0}^{1}(\Omega)$. Then, up to subsequences,

$$
\lim _{e \rightarrow 0}\left(\int_{\Omega}\left|f_{e}\right|^{2^{*}-\varepsilon} d x-\int_{\Omega}\left|f_{\varepsilon}-f\right|^{2^{*}-e} d x\right)=\int_{\Omega}|f|^{2^{*}} d x
$$


In the present case, $f_{\varepsilon}=u_{\varepsilon 2}$ and $f=u^{*}$. Hence, there exists an $o_{\varepsilon}$ such that

$$
\begin{aligned}
\int_{\Omega}\left|u_{\varepsilon 2}-u^{*}\right|^{2^{*}} d x & =\int_{\Omega}\left|u_{\varepsilon 2}\right|^{2^{*}} d x-\int_{\Omega}\left|u^{*}\right|^{2^{*}} d x+o_{\varepsilon} \\
& =1-\int_{\Omega}\left(u^{*}\right)^{2^{*}} d x+o_{\varepsilon}
\end{aligned}
$$

From (3), we derive

$$
\begin{aligned}
\int_{\Omega}\left|\nabla u_{\varepsilon 2}\right|^{2} d x \geq & C_{2 \cdot}\left(\int_{\Omega}\left|u^{*}\right|^{2^{*}} d x\right)^{2 / 2^{*}} \\
& +C_{2^{*}}\left(1-\int_{\Omega}\left(u^{*}\right)^{2^{*}} d x+o_{\varepsilon}\right)^{2 / 2^{*}}+o_{\varepsilon}
\end{aligned}
$$

and, letting $\varepsilon \rightarrow 0$, we obtain

$$
C_{2^{*}} \geq C_{2^{*}}\left(\int_{\Omega}\left|u^{*}\right|^{2^{*}} d x\right)^{2 / 2^{*}}+C_{2^{*}}\left(1-\int_{\Omega}\left(u^{*}\right)^{2^{*}} d x\right)^{2 / 2^{*}}
$$

We then use the inequality

$$
\alpha^{2 / 2^{*}}+\beta^{2 / 2^{*}} \geq(\alpha+\beta)^{2 / 2^{\circ}} \quad(\alpha, \beta \geq 0)
$$

and notice that it is strict unless $\alpha=0$ or $\beta=0$. The lower-semicontinuity of the norm of $L^{2^{*}}(\Omega)$, with respect to the weak topology of $H_{0}^{1}(\Omega)$ implies that the norm $\left\|u^{*}\right\|_{L^{2^{*}}(\Omega)}$ is smaller or equal to 1 . Hence from (5) and (6) we infer

$$
C_{2^{*}}=C_{2^{*}}\left(\int_{\Omega}\left|u^{*}\right|^{2^{*}} d x\right)^{2 / 2^{*}}+C_{2^{*}}\left(1-\int_{\Omega}\left|u^{*}\right|^{2^{*}} d x\right)^{2 / 2^{*}} .
$$

By the previous remark $\int_{\Omega}\left|u^{*}\right|^{2^{*}} d x$ is equal to 0 or 1 .' If $\left\|u^{*}\right\|_{L^{2^{*}}(\Omega)}$ were equal to $1, u^{*}$ would be a minimizer of problem (P1). This is impossible, which proves that $u^{*}=0$, almost everywhere in $\Omega$

Similarly (see [6]) one has

Lemma 2.5: Suppose that $u_{e 3}$ is a minimizer or an $o_{e}$-minimizer of problem (P3) $)_{e}$. Then the sequence $\left(u_{e 3}\right)_{e}$ converges to 0 in the weak topology of $H_{0}^{1}(\Omega)$.

Remark 2.6: Given any point $y \in \Omega$, we can always find a minimizing sequence for problem (P1) which concentrates in $y$. 


\section{Epi-convergence of sequences of functionals and applications}

Let us first recall the definition of this variational convergence in an abstract setting.

Definition 3.1 (see [1: Proposition 1.14]): Let $F_{\varepsilon}$ and $F$ be functions defined on a metric space $(X, \tau)$, with values in $\mathbb{R} \cup\{+\infty\}$. Then the sequence $\left(F_{\varepsilon}\right)_{e}$ epi-converges to $F$ in the topology $\tau$ if the two following assertions are satisfied:

(E1) $\quad \limsup _{\varepsilon \rightarrow 0} F_{e}\left(x_{\varepsilon}^{0}\right) \leq F(x)$ for all $x \in X$ and some sequence $x_{\varepsilon}^{0} \stackrel{r}{\rightarrow} x$.

(E2) $\liminf _{\varepsilon \rightarrow 0} F_{e}\left(x_{\varepsilon}\right) \geq F(x)$ for all $x \in X$ and all sequences $x_{\varepsilon} \stackrel{\tau}{\longrightarrow} x$.

This epi-convergence is a special case of the $\Gamma$-convergence introduced by De Giorgi in [9] and is well-fitted to the study of minimization problems, even in the case of non-existence of minimizers.

Definition 3.2: Let $\left(o_{\varepsilon}\right)_{e}$ be a family of positive numbers such that $\lim _{\varepsilon \rightarrow 0} o_{\varepsilon}=0$. A function $u_{\varepsilon}$ is an $o_{\varepsilon}$-minimizer of the functional $F$ defined on $X$ if it satisfies $F\left(u_{\varepsilon}\right) \leq$ $\inf _{u \in X} F(u)+o_{e}$.

Then, we have

Theorem 3.3 (see [1: Theorem 1.10]): Assume that

(1) the sequence $\left(F_{e}\right)_{e}$ epi-converges to $F$ in the topology $\tau$

(2) there exists an $o_{\varepsilon}$-minimizer $u_{\varepsilon}$ of $F_{\varepsilon}$ such that the-sequence $\left(u_{\varepsilon}\right)_{\varepsilon}$ is $\tau$-relatively compact.

Then every limit point $u$ of the sequence $\left(u_{\varepsilon}\right)_{e}$ (such that the subsequence $\left(u_{e_{k}}\right)_{e_{k}}$ converges to $u$ in the topology $\tau$.) is a minimizer of $F$ and $\lim _{k \rightarrow+\infty} F_{\varepsilon_{k}}\left(u_{e_{k}}\right)=F(u)$ :

Epi-convergence is stable with respect to continuous perturbations, since the following statement is true.

Proposition 3.4 [1: Theorem 2.15]:: Suppose that the sequence $\left(F_{\varepsilon}\right)_{\varepsilon}$ epi-converges to $F$ in the topology $\tau$ and that $G$ is $\tau$-continuous on $X$. Then the sequence $\left(F_{\varepsilon}+G\right)_{\varepsilon}$ epi-converges to $F+G$ in this topology $\tau$.

In the present case of nonlinear elliptic equations, we shall apply this variational convergence to the functionals $F_{\varepsilon 2}$ and $F_{\varepsilon 3}$ associated to the problems (P2) $)_{e}$ and (P3) $)_{e}$ respectively. Notice that these functionals $F_{\varepsilon 2}$ and $F_{e 3}$ can be defined on the whole space $H_{0}^{1}(\Omega)$ by

$$
\begin{aligned}
& F_{\varepsilon 2}(u)= \begin{cases}\int_{\Omega}|\nabla u|^{2} d x & \text { if } u \in K_{e}(\Omega) \\
+\infty & \text { if } u \in H_{0}^{1}(\Omega) \backslash K_{\varepsilon}(\Omega)\end{cases} \\
& F_{\varepsilon 3}(u)= \begin{cases}\int_{\Omega}|\nabla u|^{2} d x-\varepsilon \int_{\Omega} u^{2} d x & \text { if } u \in K(\Omega) \\
+\infty & \text { if } u \in H_{0}^{1}(\Omega) \backslash K(\Omega)\end{cases}
\end{aligned}
$$

Our main result in this paragraph is the following 
Theorem 3.5: The sequences $\left(F_{e k}\right)_{e}(k=2,3)$ epi-converge in the weak topology of $H_{0}^{1}(\Omega)$ to $F_{0}$ defined on this space by

$$
F_{0}(u)= \begin{cases}\int_{\Omega}|\nabla u|^{2} d x+C_{2^{*}}\left(1-\int_{\Omega}|u|^{2^{*}} d x\right)^{2 / 2^{*}} & \text { if } u \in H_{0}^{1}(\Omega) \cap B_{2^{*}} \\ +\infty & \text { if } u \in H_{0}^{1}(\Omega) \backslash\left(H_{0}^{1}(\Omega) \cap B_{2^{\bullet}}\right)\end{cases}
$$

where $B_{2^{*}}=\left\{\left.u \in L^{2^{*}}(\Omega)\left|\int_{\Omega}\right| u\right|^{2^{\bullet}} d x \leq 1\right\}$ is the unit closed ball of $L^{2^{\bullet}}(\Omega)$.

Proof: Let us first verify assertion $(\mathrm{E} 1)$ for the sequence $\left(F_{\varepsilon 2}\right)_{\varepsilon}$ :

$\lim \sup _{\varepsilon \rightarrow 0} F_{\varepsilon 2}\left(v_{\varepsilon}^{0}\right) \leq F_{0}(v)$ for all $v \in H_{0}^{1}(\Omega)$ and some sequence $v_{\varepsilon}^{0} \stackrel{\tau}{\longrightarrow} v$

where $\tau$ represents the weak topology of the Hilbert space $H_{0}^{1}(\Omega)$ (notice that $\tau$ can be regarded as a metric on the bounded subsets of this space).

For every $v \in H_{0}^{1}(\Omega) \cap B_{2^{*}}$, we define

$$
v_{\varepsilon}^{0}=\frac{v-\beta u_{e 2}}{\left\|v-\beta u_{e 2}\right\|_{L^{2-*}(\Omega)}} \quad \text { with } \beta=\left(1-\int_{\Omega}|v|^{2^{\bullet}} d x\right)^{1 / 2 *}
$$

where $u_{\varepsilon 2}$ is a minimizer of $C_{\varepsilon 2}(\Omega)$. Lemma 2.4 and (4) imply that the sequence $\left(v_{e}^{0}\right)_{e}$ converges to $v$ in the weak topology of $H_{0}^{1}(\Omega)$. Indeed, since the sequence $\left(u_{\varepsilon 2}\right)_{\varepsilon}$ converges to 0 in the weak topology of this space, we deduce from (4)

$$
\int_{\Omega}\left|v-\beta u_{\varepsilon 2}\right|^{2^{*}-\varepsilon} d x-\beta^{2^{*}-\varepsilon} \int_{\Omega}\left|u_{\varepsilon 2}\right|^{2^{*}-\varepsilon} d x=\int_{\Omega}|v|^{2^{*}} d x+o_{\varepsilon}
$$

which implies

$$
\lim _{e \rightarrow 0} \int_{\Omega}\left|v-\beta u_{e 2}\right|^{*^{*}-\varepsilon} d x=1-\int_{\Omega}|v|^{2^{*}} d x+\int_{\Omega}|v|^{2^{*}} d x=1
$$

and then that the sequence $\left(v_{\varepsilon}^{0}\right)_{\varepsilon}$ converges to $v$ in the weak topology of $H_{0}^{1}(\Omega)$. Lemma 2.4 implies that

$$
\begin{aligned}
\limsup _{\varepsilon \rightarrow 0} F_{\varepsilon 2}\left(v_{e}^{0}\right)= & \int_{\Omega}|\nabla v|^{2^{\bullet}} d x+C_{2 \bullet} \beta^{2} \int_{\Omega}|\nabla v|^{2^{\bullet}} d x \\
& +C_{2 \cdot}\left(1-\int_{\Omega}|v|^{2^{\bullet}} d x\right)^{2 / 2^{\bullet}} \\
= & F_{0}(v) .
\end{aligned}
$$

Finally, since the embedding $H_{0}^{1}(\Omega) \hookrightarrow L^{2^{*}}(\Omega)$ is continuous, we deduce that every element $v \in H_{0}^{1}(\Omega)$ such that $\|v\|_{L^{2^{*}}(\Omega)}>1$ cannot be the limit, in the weak topology of $H_{0}^{1}(\Omega)$; of a sequence $\left(v_{\varepsilon}^{0}\right)_{e}$ of functions belonging to $H_{0}^{1}(\Omega) \cap B_{2} \cdot$. This proves that $F_{0}(v)=+\infty$ for every $v \in H_{0}^{1}(\Omega)$ such that $\|v\|_{L^{2^{*}}(\Omega)}>1$.

Let us verify assertion (E2) for the sequence $\left(F_{e 2}\right)_{e}$, i.e. that 
$\liminf _{e \rightarrow 0} F_{e 2}\left(v_{\varepsilon}\right) \geq F_{0}(v)$ for every $v \in H_{0}^{1}(\Omega)$ and every sequence $v_{\varepsilon} \stackrel{\tau}{\longrightarrow} v$.

We may assume that $v \in B_{2}$. and that $v_{\varepsilon} \in S_{2^{*}-\varepsilon}$ for every $\varepsilon$. Indeed, otherwise assertion (E2) is trivially satisfied. By (3) and (4), we obtain

$$
\liminf _{\varepsilon \rightarrow 0} \int_{\Omega}\left|\nabla v_{\varepsilon}\right|^{2} d x \geq \int_{\Omega}|\nabla v|^{2} d x+C_{2^{\bullet}}\left(1-\int_{\Omega}|v|^{2^{\bullet}} d x\right)^{2 / 2^{*}}=F_{0}(v)
$$

This completes the proof for the sequence $\left(F_{\varepsilon 2}\right)_{\varepsilon}$.

Let us now give the main ideas of the proof of the corresponding result for the sequence $\left(F_{e 3}\right)_{e}$. We introduce the functional $F$ defined on $H_{0}^{1}(\Omega)$ by

$$
F(u)= \begin{cases}\int_{\Omega}|\nabla u|^{2} d x & \text { if } u \in K(\Omega) \\ +\infty & \text { if } u \in H_{0}^{1}(\Omega) \backslash K(\Omega)\end{cases}
$$

and notice that $F_{e 3}$ is equal to $F(\cdot)-\varepsilon\|\cdot\|_{L^{2}(\Omega)}^{2}$. From the compact embedding $H_{0}^{1}(\Omega) \hookrightarrow$ $L^{2}(\Omega)$, one easily infers that $\left(F_{e 3}\right)_{\varepsilon}$ epi-converges to some $F_{0}$ in the weak topology of $H_{0}^{1}(\Omega)$ if and only if the constant sequence the members of which are $F$, epi-converges to the same $F_{0}$, in the weak topology of $H_{0}^{1}(\Omega)$. The main modification for proving assertion (E1) in this case consists in the introduction of the test function

$$
v_{\varepsilon}^{0}=\frac{v-\beta u_{\varepsilon 3}}{\left\|v-\beta u_{e 3}\right\|_{L^{2^{*}}(\Omega)}} \quad \text { with } \quad \beta=\left(1-\int_{\Omega}|v|^{2^{*}} d x\right)^{1 / 2^{*}}
$$

where $u_{e 3}$ is a minimizer or an $o_{e}$-minimizer of $C_{e 3}(\Omega)$ (see [6] for the detailed proof)

Remark 3.6: Because of Proposition 3.4, one can replace in Theorem 3.5 the functionals $F_{e 2}$ and $F_{\varepsilon 3}$ by

$$
F_{\varepsilon 2}(\cdot)-\int_{\Omega} G(x, \cdot) d x \quad \text { and } \quad F_{e 3}(\cdot)-\int_{\Omega} G(x, \cdot) d x
$$

respectively, where the functional $u \mapsto \int_{\Omega} G(x, u) d x$ is continuous with respect to the weak topology of $H_{0}^{1}(\Omega)$. This leads to the following perturbation result.

Corollary 3.7: Assume that $u \mapsto \int_{\Omega} G(x, u) d x$ is continuous for the weak topology of $H_{0}^{1}(\Omega)$. Then the sequences

$$
\left(F_{e k}-\int_{\Omega} G(x, \cdot) d x\right)_{e} \quad(k=2,3)
$$

epi-converge in the weak topology of $H_{0}^{1}(\Omega)$ to the functional $F_{0}-\int_{\Omega} G(x, \cdot) d x$. 


\section{Further results concerning the behaviour of $\left(u_{\varepsilon 2}\right)_{\varepsilon}$ minimizers of problem (P2).}

The purpose of this section is to study, by means of epi-convergence methods, the behaviour of the sequence $\left(u_{\varepsilon 2}\right)_{e}$, where $u_{e 2}$ is the solution of the variational problem $(\mathrm{P} 2)_{e}$, when $\varepsilon \rightarrow 0$.

From the classical regularity theory for the solutions of elliptic equations, one deduces that the minimizer $u_{\varepsilon 2}$ of problem $(\mathrm{P} 2)_{e}$ or the solution of (2), belongs to $C^{1}(\bar{\Omega}) \cap C^{2}(\Omega)$. Hence, there exists $x_{\varepsilon} \in \bar{\Omega}$ such that $u_{\varepsilon 2}$ achieves its maximum in $x_{\varepsilon}$. Applying the methods of [8] and [10], one proves that $x_{e}$ stays away from the boundary $\partial \Omega$ of $\Omega($ see [11]):

$$
\operatorname{dist}\left(x_{e}, \partial \Omega\right) \geq \delta \text { for some } \delta>0 \text { and all } \varepsilon>0 .
$$

Han has proved in [11] that $u_{e 2}$ "blows up in $x_{\varepsilon}$ ", when $\varepsilon \rightarrow 0$, that is

$$
\lim _{\varepsilon \rightarrow 0}\left\|u_{e 2}\right\|_{L^{\infty}(\Omega)}=\lim _{\varepsilon \rightarrow 0} u_{e 2}\left(x_{\varepsilon}\right)=+\infty
$$

(see also [15: Prop. 2]). The next result shows how this function $u_{\dot{ }}$ blows up in the $H_{0}^{1}$-norm.

Theorem 4.1: Let $\alpha_{N}, \mu_{N}, \mu_{\varepsilon}$ and $w_{\varepsilon}$ be defined as

$$
\begin{aligned}
\left(\alpha_{N}\right)^{2^{*}} & =\pi^{N / 2} \frac{\Gamma(N / 2)}{\Gamma(N)}, \quad \mu_{N}=\left(\alpha_{N}\right)^{-2 /(N-2)} \\
\mu_{\varepsilon} & =\frac{\mu_{N}}{\left\|u_{\varepsilon 2}\right\|_{L^{\infty}(\Omega)}^{2 /(N-2)}}, \quad w_{e}(y)=\frac{1}{\alpha_{N}}\left(\frac{\mu_{\varepsilon}}{\mu_{\varepsilon}^{2}+\left|y-x_{0}\right|^{2}}\right)^{(N-2) / 2} \quad\left(y \in \mathbb{R}^{N}\right)
\end{aligned}
$$

with $\lim _{e \rightarrow 0} x_{e}=x_{0}$. Then

$$
\int_{\mathbb{R}^{N}}\left|\nabla\left(Q u_{\varepsilon 2}-w_{e}\right)\right|^{2} d y \rightarrow 0 \quad \text { as } \varepsilon \rightarrow 0
$$

where $Q$ denotes the extension operator (by 0$)$ from $H_{0}^{1}(\Omega)$ into $H_{0}^{1}\left(\mathbb{R}^{N}\right)$.

Proof: Let us first remark the following change of scale suggested in [11]:

$$
\begin{aligned}
\Omega_{\varepsilon 2} & =\left\|u_{\varepsilon 2}\right\|_{L^{\infty}(\Omega)}^{\left(2^{*}-\varepsilon\right) / N}\left(\Omega-x_{\varepsilon}\right) \\
v_{e 2}(y) & =\frac{1}{\left\|u_{\varepsilon 2}\right\|_{L^{\infty}(\Omega)}} u_{e 2}\left(\frac{1}{\left\|u_{\varepsilon 2}\right\|_{L^{\infty}(\Omega)}^{\left(2^{\bullet}-\varepsilon\right) / N}} y+x_{\varepsilon}\right) \quad\left(y \in \Omega_{\varepsilon 2}\right) .
\end{aligned}
$$

Notice that (7) implies that this open set $\Omega_{\varepsilon 2}$ increases to $\mathbb{R}^{N}$, when $\varepsilon \rightarrow 0$. Moreover, $v_{\varepsilon 2}$ is the solution of the minimization problem associated to the functional $\tilde{F}_{\varepsilon 2}$ defined on $H_{0}^{1}\left(\Omega_{e 2}\right)$ by

$$
\tilde{F}_{e 2}(v)= \begin{cases}\int_{\Omega_{e 2}}|\nabla v|^{2} d y & \text { if } v \in K_{e}\left(\Omega_{\varepsilon 2}\right) \\ +\infty & \text { if } v \in H_{0}^{1}\left(\Omega_{\varepsilon 2}\right) \backslash K_{\varepsilon}\left(\Omega_{\varepsilon 2}\right)\end{cases}
$$


A straightforward computation (cf. Section 1) yields

$$
\begin{aligned}
& -\Delta v_{\varepsilon 2}=\left(\int_{\Omega_{\varepsilon 2}}\left|\nabla v_{\varepsilon 2}\right|^{2} d y\right) v_{\varepsilon 2}^{2^{*}-\varepsilon-1} \quad \text { in } \Omega_{\varepsilon 2} \\
& \int_{\Omega_{\varepsilon 2}}\left|v_{\varepsilon 2}\right|^{2^{*}-\varepsilon} d y=1 \quad\left(v_{\varepsilon 2}>0 \text { in } \Omega_{\varepsilon 2}, v_{\varepsilon 2} \in H_{0}^{1}\left(\Omega_{\varepsilon 2}\right)\right)
\end{aligned}
$$

and $v_{e 2}$ satisfies $0 \leq v_{\varepsilon 2}(y) \leq 1, v_{e 2}(0)=1$

The behaviour of the sequence $\left(v_{\varepsilon 2}\right)_{\varepsilon}$ when $\varepsilon \rightarrow 0$ will be derived from the following

Lemma 4.2: The behaviour of the functionals $F_{e 2}$ and their minimizers as $\varepsilon \rightarrow 0$ is described as follows.

(i) The sequence $\left(\tilde{F}_{\varepsilon 2}\right)_{e}$ epi-converges in the strong topology of $H_{0}^{1}\left(\mathbb{R}^{N}\right)$ to the functional $\tilde{F}_{0}$ defined by

$$
\tilde{F}_{0}(v)= \begin{cases}\int_{\mathbb{R}^{N}}|\nabla v|^{2} d y & \text { if } v \in K\left(\mathbb{R}^{N}\right) \\ +\infty & \text { if } v \in H_{0}^{1}\left(\mathbb{R}^{N}\right) \backslash K\left(\mathbb{R}^{N}\right)\end{cases}
$$

(ii) Let $Q_{\varepsilon}$ be the extension operator from $H_{0}^{1}\left(\Omega_{\varepsilon 2}\right)$ into $H_{0}^{1}\left(\mathbb{R}^{N}\right)$. Then the sequence $\left(Q_{\varepsilon} v_{\varepsilon 2}\right)_{\varepsilon}$ converges in the strong topology of $H_{0}^{1}\left(\mathbb{R}^{N}\right)$ to $V$, defined by

$$
V(y)=\frac{1}{\alpha_{N}}\left(\frac{\mu_{N}}{\mu_{N}^{2}+|y|^{2}}\right)^{(N-2) / 2} .
$$

(iii) One has

$$
\lim _{\varepsilon \rightarrow 0}\left\|u_{\varepsilon 2}\right\|_{L^{\infty}(\Omega)}^{\varepsilon}=\frac{1}{C_{2^{*}}} \int_{\mathbb{R}^{N}}|\nabla V|^{2} d y=1
$$

Proof: (i) For every $v \in K\left(\mathbb{R}^{N}\right)$, we consider the solution $v_{\varepsilon 0}$ of the problem

$$
-\Delta v_{\varepsilon 0}(y)=-\Delta v(y) \quad \text { for all } y \in \Omega_{\varepsilon 2}, \quad v_{\varepsilon 0} \in H_{0}^{1}\left(\Omega_{\varepsilon 2}\right) \text {. }
$$

We can prove that the sequence $\left(Q_{\varepsilon} v_{\varepsilon 0}\right)_{\varepsilon}$ converges to $v$ in the strong topology of $H_{0}^{1}\left(\mathbb{R}^{N}\right)$. Hence the sequence $\left(\left\|v_{\varepsilon 0}\right\|_{L^{2^{*}-\Omega_{(2)}}\left(\Omega_{\varepsilon^{2}}\right)}\right)_{e}$ converges to $\|v\|_{L^{2^{*}}\left(\mathbb{R}^{N}\right)}=1$. This implies that the sequence

$$
\left(Q_{\varepsilon}\left(\frac{v_{e 0}}{\left\|v_{\varepsilon 0}\right\|_{L^{2 *-\epsilon}\left(\Omega_{\varepsilon 2}\right)}}\right)\right)_{\epsilon}
$$

converges to $v$ in the strong topology of $H_{0}^{1}\left(\mathbb{R}^{N}\right)$ and

$$
\begin{aligned}
\underset{\varepsilon \rightarrow 0}{\limsup } \tilde{F}_{\varepsilon 2}\left(\left\|v_{\varepsilon 0}\right\|_{L^{2^{*}-\varepsilon}\left(\Omega_{\varepsilon 2}\right)}^{-1} v_{\varepsilon 0}\right) & =\underset{\varepsilon \rightarrow 0}{\limsup }\left\|v_{\varepsilon 0}\right\|_{L^{2^{*}-\varepsilon}\left(\Omega_{\varepsilon 2}\right)}^{-2} \int_{\mathbb{R}^{N}}\left|\nabla Q_{\varepsilon} v_{\varepsilon 0}\right|^{2} d x \\
& =\int_{\mathbb{R}^{N}}|\nabla v|^{2} d y=\tilde{F}_{0}(v) .
\end{aligned}
$$


Let the sequence $\left(v_{e}\right)_{e}$ converge to $v$ in the strong topology of $H_{0}^{1}\left(R^{N}\right)$. We may assume that, for every $\varepsilon,\left\|v_{\varepsilon}\right\|_{L^{2 *-\bullet}(\Omega)}$ is equal to 1 . Then

$$
\liminf _{\varepsilon \rightarrow 0} \tilde{F}_{\varepsilon 2}\left(Q_{\varepsilon} v_{\varepsilon}\right)=\underset{\varepsilon \rightarrow 0}{\limsup } \int_{\mathbb{R}^{N}}\left|\nabla Q_{\varepsilon} v_{\varepsilon}\right|^{2} d y=\int_{\mathbb{R}^{N}}|\nabla v|^{2} d y
$$

(ii) From Theorem 3.3, one infers that the sequence $\left(Q_{e} v_{e 2}\right)_{e}$ converges in the strong topology of $H_{0}^{1}\left(\mathbb{R}^{N}\right)$ to the minimizer $V$ of $\tilde{F}_{0}$, which satisfies $0 \leq V(y) \leq 1$ and $V(0)=1$, since the sequence $\left(Q_{e} v_{e 2}\right)_{e}$ converges to $V$ uniformly on compact subsets of $\mathbb{R}^{N}$. This minimizer is unique [7: Corollary 8.2] and is given by the expression indicated in (ii).

(iii) Let us compute

$$
\int_{\Omega_{\varepsilon 2}}\left|\nabla v_{e 2}\right|^{2} d y=\left\|u_{\varepsilon 2}\right\|_{L^{\infty}(\Omega)}^{e(2-N) / N} \int_{\Omega}\left|\nabla u_{e 2}\right|^{2} d x
$$

By Lemma 4.2/(ii) and (4), we have

$$
\lim _{\varepsilon \rightarrow 0}\left\|u_{\varepsilon 2}\right\|_{L^{\infty}(\Omega)}^{e(2-N) / N}=\frac{1}{C_{2} \cdot} \lim _{\varepsilon \rightarrow 0} \int_{\mathbb{R}^{N}}\left|\nabla Q_{\varepsilon} v_{\varepsilon 2}\right|^{2} d y=\frac{1}{C_{2^{*}}} \int_{\mathbb{R}^{N}}|\nabla V|^{2} d y=1
$$

This result is similar to Corollary 1 of [11]

Let us now end the

Proof of Theorem 4.1: Lemma 4.2/(ii) has established that

$$
\lim _{e \rightarrow 0} \int_{\mathbb{R}^{N}}\left|\nabla\left(Q_{e} v_{e 2}-V\right)\right|^{2} d y=0
$$

Lemma $4.2 /(\mathrm{iii})$ and the change of scale,

$$
y=\left\|u_{\varepsilon 2}\right\|_{L^{\infty}(\Omega)}^{\left(2^{*}-e\right) / N}\left(x-x_{\varepsilon}\right)
$$

associated to (8), in the preceding integral imply

$$
\lim _{\varepsilon \rightarrow 0}\left\|u_{e 2}\right\|_{L^{\infty}(\Omega)}^{-e(N-2) / N} \int_{\mathbb{R}^{N}}\left|\nabla\left(Q u_{e 2}-w_{e}\right)\right|^{2} d y=0
$$

This, together with Lemma 4.2/(iii), concludes the proof

Remark 4.3: 1 . The sequence $\left(x_{\varepsilon}\right)_{e}$ being bounded converges to some $x \in \Omega$. It is known from [11: Theorem 1] and [15: Theorem 4] that this $x$ is a critical point of $H(x, x)$. 2. Notice that from Theorem 4.1 we can recover Han's convergence results. 3. The same arguments apply to the sequence $\left(u_{\varepsilon 3}\right)_{e}$ corresponding to problem $(\mathrm{P} 3)_{e}$. 


\section{References}

[1] Attouch, H.: Variational convergence for functions and operators. London: Pitman 1984.

[2] Bandle, C.: Isoperimetric inequalities and their applications. London: Pitman 1980.

[3] Bandle, C. and M. Flucher: Conformal radius, Robin's problem and Liouville's equation. In preparation.

[4] Brezis, H. and E. Lieb: A relation between pointwise convergence of functions and convergence of functionals. Proc. Amer. Math. Soc. 88 (1983), 486 - 490.

[5] Brezis, H. and L. Nirenberg: Positive solutions of nonlinear elliptic equations involving critical Sobolev exponents. Comm. Pure Appl. Math. 36 (1983), 437 - 477.

[6] Brillard, A.: Analyse limite par épi-convergence de trois problèmes non-linéaires avec exposont critique de Sobolev. Preprint. Mulhouse: : Université de Haute-Alsace, Preprint No. 54 (1993), pp. 1 - 29.

[7] Caffarelli, L., Gidas, B. and J. Spruck: Asymptotic symmetry and local behaviour of semilinear elliptic equations with critical growth. Comm. Pure Appl. Math. 42 (1989), $271-297$.

[8] De Figueiredo, D. G., Lions, P. L. and R. D. Nussbaum: A priori estimates and existence of positive solutions of semilinear elliptic equations. J. Math. Pures Appl. 61 (1982), 41 $-63$.

[9] De Giorgi, E.: Convergence problems for functionals and operators. In: Proc. Int. Meeting on Recent Methods in Nonlinear Analysis, Rome 1978 (eds.: E. De Giorgi et al). Bologna: Pitagora Editrice 1979, pp. 131 - 188.

[10] Gidas, B., Ni, W. and L. Nirenberg: Symmetry and related properties via the maximum principle. Comm. Math. Phys. 68 (1979), $209 \cdot 243$.

[11] Zheng-Chao Han: Asymptotic approach to singular solutions for nonlinear elliptic equations involving critical Sobolev exponent. Ann. Inst. Henri Poincaré 8 (1991), 159 174.

[12] Lions, P. L.: Applications de la méthode de concentration-compacité à l'existence de fonctions extrémales. C.R.A.S. (Serie 1) 296 (1983), 645 - 648.

[13] Lions, P. L.: The concentration-compactness principle in the Calculus of Variations. The limit case. Parts 1 and 2. Riv. Mat. Iberoamericana 1 (1985)1, $145-201$ and 1(1985)2, $45-121$.

[14] Pohozaev, S. I.: Eigenfunctions of the equation $\Delta u+\lambda f(u)=0$ (in Russian). Dokl. Akad. Nauk SSSR 165 (1965), 36 - 39. Engl. transl.: Sov. Math. 6 (1965), 1408 - 1411.

[15] Rey, O.: The role of the Green's function in a non-linear elliptic equation involving the critical Sobolev exponent. J. Func. Anal. 89 (1990), 1 - 52.

[16] Struwe, M.: Variational methods. Heidelberg: Springer-Verlag 1990.

Received 13.01.1994. 